\title{
COMPACT SUMS OF TOEPLITZ PRODUCTS AND TOEPLITZ ALGEBRA ON THE DIRICHLET SPACE
}

\author{
YOUNG JOO LEE
}

(Received August 4, 2014, revised October 21, 2014)

\begin{abstract}
In this paper we consider Toeplitz operators on the Dirichlet space of the ball. We first characterize the compactness of operators which are finite sums of products of two Toeplitz operators. We also characterize Fredholm Toeplitz operators and describe the essential norm of Toeplitz operators. By using these results, we establish a short exact sequence associated with the $C^{*}$-algebra generated by all Toeplitz operators.
\end{abstract}

1. Introduction. Let $B=B_{n}$ be the unit ball in the complex $n$-space $\mathbb{C}^{n}$ and $V$ be the Lebesgue volume measure on $B$ normalized so that $V(B)=1$. The Sobolev space $\mathscr{S}$ is the completion of the space of all smooth functions $f$ on $B$ for which

$$
\|f\|=\left\{\left|\int_{B} f d V\right|^{2}+\int_{B}\left(|\mathcal{R} f|^{2}+|\widetilde{\mathcal{R}} f|^{2}\right) d V\right\}^{1 / 2}<\infty
$$

where

$$
\mathcal{R} f(z)=\sum_{i=1}^{n} z_{i} \frac{\partial f}{\partial z_{i}}(z), \quad \widetilde{\mathcal{R}} f(z)=\sum_{i=1}^{n} \overline{z_{i}} \frac{\partial f}{\partial \overline{z_{i}}}(z)
$$

for $z=\left(z_{1}, \ldots, z_{n}\right) \in B$. Then the Sobolev space $\mathscr{S}$ is a Hilbert space with respect to the inner product

$$
\langle f, g\rangle=\int_{B} f d V \overline{\int_{B} g d V}+\int_{B}(\mathcal{R} f \overline{\mathcal{R} g}+\widetilde{\mathcal{R}} f \overline{\widetilde{\mathcal{R}} g}) d V .
$$

The Dirichlet space $\mathscr{D}$ is then a subspace of $\mathscr{S}$ consisting of all holomorphic functions on $B$. Then it is easy to see that the Dirichlet space $\mathscr{D}$ is closed in $\mathscr{S}$. We let $P$ be the Hilbert space orthogonal projection from $\mathscr{S}$ onto $\mathscr{D}$. Put

$$
\mathscr{L}^{1, \infty}=\left\{\varphi \in \mathscr{S}: \varphi, \frac{\partial \varphi}{\partial z_{j}}, \frac{\partial \varphi}{\partial \bar{z}_{j}} \in L^{\infty}, j=1, \ldots, n\right\}
$$

where the derivatives are taken in the sense of distribution. By the Sobolev embedding theorem([1, Theorem 5.4]), each function in $\mathscr{L}^{1, \infty}$ can be extended to a continuous function on the closed unit ball $\bar{B}$. Thus we will use the same notation between a function in $\mathscr{L}^{1, \infty}$ and its continuous extension to $\bar{B}$. Also, for $\varphi \in \mathscr{L}^{1, \infty}$, we note that $\mathcal{R} \varphi, \widetilde{\mathcal{R}} \varphi \in L^{\infty}$.

2010 Mathematics Subject Classification. Primary 47B35; Secondary 32A37.

Key words and phrases. Toeplitz operator, Dirichlet space, Fredholm operators, Toeplitz algebra.

This research was supported by Basic Science Research Program through the National Research Foundation of Korea(NRF) funded by the Ministry of Education(NRF-2014R1A1A4A01003810). 
Given $u \in \mathscr{L}^{1, \infty}$, the Toeplitz operator $T_{u}$ with symbol $u$ is defined on $\mathscr{D}$ by

$$
T_{u} f=P(u f)
$$

for functions $f \in \mathscr{D}$. Then $T_{u}$ is a bounded linear operator on $\mathscr{D}$; see Proposition 1 of Section 3.

In this paper we first consider operators which are finite sums of products of two Toeplitz operators. More explicitly, we consider operators $L$ of the form

$$
L=\sum_{j=1}^{N} T_{u_{j}} T_{v_{j}},
$$

where $u_{j}, v_{j} \in \mathscr{L}^{1, \infty}$. We then study the problem of when such an operator of the form (1) is compact on $\mathscr{D}$. On the setting of the smaller Dirichlet space $\mathscr{D}_{0}$ consisting of all holomorphic functions $f$ in $\mathscr{S}$ which have restriction $f(0)=0$, the same problem has been studied in [10] or [12] on the unit disk and in [11] on the unit ball. But we mention that the arguments used there do not work on our Dirichlet space $\mathscr{D}$.

By using a completely different argument, we prove in this paper that an operator of form (1) is compact on $\mathscr{D}$ if and only if the corresponding sum of products of symbols is equal to 0 on $\partial B$, the boundary of $B$; see Theorem 7. Specially, the case of $N=1$ in (1) reduces the compact product problem of when a product of two Toeplitz operators is compact. As an application of Theorem 7, we show that for pluriharmonic symbols, there is no nontrivial compact product for $n \geq 2$ but there are many for $n=1$. Such higher dimensional phenomena on $\mathscr{D}_{0}$ has been also noticed in [11].

More specially, for a single Toeplitz operator, the corresponding problem will be of particular interest. On the smaller Dirichlet space $\mathscr{D}_{0}$ of the unit disk, it is known that for a single Toeplitz operator, it is compact on $\mathscr{D}_{0}$ if and only if it equals 0 on $\mathscr{D}_{0}$; see Proposition 3.1 and Theorem 3.4 of [12]. On the contrary to this result on $\mathscr{D}_{0}$, our result shows that there exists a nonzero compact Toeplitz operator on our Dirichlet space $\mathscr{D}$; see Corollary 8 and its remark. These results will be collected in Section 3.

Next, in Section 4, we first characterize Fredholm Toeplitz operators and describe the essential norm of Toeplitz operators on $\mathscr{D}$. We then study the Toeplitz algebra $\mathcal{T}$ generated by all Toeplitz operators. By using a consequence of Theorem 7 , we show that there is a short exact sequence

$$
0 \rightarrow \mathcal{K} \rightarrow \mathcal{T} \rightarrow C(\partial B) \rightarrow 0
$$

where $\mathcal{K}$ is the algebra of all compact operators on $\mathscr{D}$ and $C(\partial B)$ is the algebra of all continuous functions on $\partial B$; see Theorem 16. On the smaller Dirichlet space $\mathscr{D}_{0}$ of the unit disk, the corresponding characterizations have been obtained in [2] where a bit different Toeplitz operators have been considered.

2. Preliminaries. In this section we introduce the well known Bergman space and then investigate some useful connection between projections on the Bergman space and Dirichlet space. 
For any multi-index $\alpha=\left(\alpha_{1}, \ldots, \alpha_{n}\right)$ where each $\alpha_{k}$ is a nonnegative integer, we will write $|\alpha|=\alpha_{1}+\cdots+\alpha_{n}$ and $\alpha !=\alpha_{1} ! \cdots \alpha_{n}$ !. We will also write

$$
z^{\alpha}=z_{1}^{\alpha_{1}} \cdots z_{n}^{\alpha_{n}}
$$

for $z=\left(z_{1}, \ldots, z_{n}\right) \in B$. Note that $\mathcal{R} z^{\alpha}=|\alpha| z^{\alpha}$ for every multi-index $\alpha$.

We let $L^{2}=L^{2}(B, V)$ be the usual Lebesgue space and $A^{2}$ be the well known Bergman space consisting of all holomorphic functions in $L^{2}$. Let $Q$ be the Bergman projection which is the orthogonal projection from $L^{2}$ onto $A^{2}$ whose its explicit formula can be written as

$$
Q \psi(z)=\int_{B} \psi(w) \overline{B_{z}(w)} d V(w), \quad z \in B
$$

for functions $\psi \in L^{2}$. Here $B_{z}$ is the Bergman kernel given by

$$
B_{z}(w)=\frac{1}{(1-w \cdot \bar{z})^{n+1}}, \quad w \in B
$$

where $w \cdot \bar{z}=w_{1} \bar{z}_{1}+\cdots+w_{n} \bar{z}_{n}$ is the Hermitian inner product for points $z, w \in \mathbb{C}^{n}$. Since

$$
B_{z}(w)=\sum_{|\alpha| \geq 0} \frac{(n+|\alpha|) !}{n ! \alpha !} \overline{z^{\alpha}} w^{\alpha}, \quad z, w \in B
$$

we have

$$
Q \psi(z)=\sum_{|\alpha| \geq 0} \frac{(n+|\alpha|) !}{n ! \alpha !} z^{\alpha} \int_{B} \overline{w^{\alpha}} \psi(w) d V(w), \quad z \in B
$$

for functions $\psi \in L^{2}$; see Chapter 2 of [14] for details and related facts.

Each point evaluation is easily verified to be a bounded linear functional on $\mathscr{D}$. Hence, for each $z \in B$, there exists a unique kernel function $K_{z} \in \mathscr{D}$ which has the following reproducing property:

$$
f(z)=\left\langle f, K_{z}\right\rangle
$$

for functions $f \in \mathscr{D}$. Since

$$
\int_{B}\left|z^{\alpha}\right|^{2} d V(z)=\frac{n ! \alpha !}{(n+|\alpha|) !}
$$

we have

$$
\| z^{\alpha}||^{2}=|\alpha|^{2} \int_{B}\left|z^{\alpha}\right|^{2} d V(z)=\frac{n !|\alpha|^{2} \alpha !}{(n+|\alpha|) !}
$$

for each multi-index $\alpha$ with $|\alpha|>0$; see Lemma 1.11 of [14]. Note that the $\operatorname{set}\left\{z^{\alpha}:|\alpha| \geq 0\right\}$ spans a dense subset of $\mathscr{D}$. Thus it can be easily seen that the kernel function $K_{z}$ on $\mathscr{D}$ has the following explicit formula

$$
K_{z}(w)=1+\sum_{|\alpha|>0} \frac{(n+|\alpha|) !}{n !|\alpha|^{2} \alpha !} \overline{z^{\alpha}} w^{\alpha}
$$


for $z, w \in B$. Since $K_{z}(0)=1$ for all $z \in B$, it follows from the reproducing property and (3) that

$$
\begin{aligned}
P \psi(z) & =\left\langle\psi, K_{z}\right\rangle \\
& =\int_{B} \psi d V+\int_{B} \mathcal{R} \psi \overline{\mathcal{R} K_{z}} d V \\
& =\int_{B} \psi d V+\sum_{|\alpha|>0} \frac{(n+|\alpha|) !}{n !|\alpha| \alpha !} z^{\alpha} \int_{B} \overline{w^{\alpha}} \mathcal{R} \psi(w) d V(w), \quad z \in B
\end{aligned}
$$

for functions $\psi \in \mathscr{S}$. Combining the above with (2), we can see

$$
\begin{aligned}
\mathcal{R}(P \psi)(z) & =\sum_{|\alpha|>0} \frac{(n+|\alpha|) !}{n ! \alpha !} z^{\alpha} \int_{B} \overline{w^{\alpha}} \mathcal{R} \psi(w) d V(w) \\
& =Q(\mathcal{R} \psi)(z)-Q(\mathcal{R} \psi)(0), \quad z \in B
\end{aligned}
$$

for functions $\psi \in \mathscr{S}$.

3. Compact sums of Toeplitz products. In this section, we consider operators having the form of finite sums of products of two Toeplitz operators and then characterize such an operator to be compact on $\mathscr{D}$. In the following, we use the notation

$$
\|f\|_{2}=\left(\int_{B}|f|^{2} d V\right)^{\frac{1}{2}}
$$

for functions $f \in L^{2}$. Note that $|f(0)| \leq\|f\|_{2}$ for all $f \in A^{2}$; see Theorem 2.1 of [14] for details.

We start with the boundedness of Toeplitz operators with symbol in $\mathscr{L}^{1, \infty}$.

Proposition 1. For $u \in \mathscr{L}^{1, \infty}$, the Toeplitz operator $T_{u}$ is bounded on $\mathscr{D}$.

Proof. Let $f \in \mathscr{D}$ be an arbitrary function. Note that $u f \in \mathscr{S}$ and

$$
\|f\|_{2} \leq\|\mathcal{R} f\|_{2} \leq\|f\| .
$$

Hence

$$
|P(u f)(0)|=\left|\int_{B} u f d V\right| \leq\|u\|_{\infty}\|f\|_{2} \leq\|u\|_{\infty}\|f\| .
$$

Also, by (5) and the $L^{2}$-boundedness of the Bergman projection $Q$, we see

$$
\begin{aligned}
\|\mathcal{R}(P(u f))\|_{2} & \leq\|Q(\mathcal{R}(u f))\|_{2}+|Q(\mathcal{R}(u f))(0)| \\
& \leq 2\|Q(\mathcal{R}(u f))\|_{2} \\
& \leq 2\|\mathcal{R}(u f)\|_{2} \\
& =2\|f \mathcal{R} u+u \mathcal{R} f\|_{2} \\
& \leq 2\left(\|\mathcal{R} u\|_{\infty}+\|u\|_{\infty}\right)\|f\| .
\end{aligned}
$$


Combining the above with (7), we see that there exists a constant $C$ depending only on $u$ such that

$$
\left\|T_{u} f\right\|^{2}=\|P(u f)\|^{2}=|P(u f)(0)|^{2}+\|\mathcal{R}(P(u f))\|_{2}^{2} \leq C\|f\|^{2}
$$

for every $f \in \mathscr{D}$, which implies the boundedness of $T_{u}$ as desired. The proof is complete.

For each $a \in B$, we let

$$
E_{a}(z)=\mathcal{R} K_{a}(z)=\sum_{|\alpha|>0} \frac{(n+|\alpha|) !}{n !|\alpha| \alpha !} \overline{a^{\alpha}} z^{\alpha}, \quad z \in B .
$$

Since $\mathcal{R} E_{a}=B_{a}-1$, we have

$$
\left\|E_{a}\right\|^{2}=\left\|\mathcal{R} E_{a}\right\|_{2}^{2}=B_{a}(a)-1=\frac{1-\left(1-|a|^{2}\right)^{n+1}}{\left(1-|a|^{2}\right)^{n+1}}
$$

for all $a \in B$. Since $\left\|B_{a}\right\|_{2}=\left(1-|a|^{2}\right)^{-\frac{n+1}{2}}$ for all $a \in B$, we have

$$
\lim _{|a| \rightarrow 1} \frac{\left\|B_{a}\right\|_{2}}{\left\|E_{a}\right\|}=1 \text {. }
$$

In the following, we put

and use the notation

$$
e_{a}(z):=\frac{1}{\left\|E_{a}\right\|} E_{a}(z), \quad a, z \in B
$$

$$
\langle\varphi, \psi\rangle_{2}=\int_{B} \varphi \bar{\psi} d V
$$

for functions $\varphi, \psi \in L^{2}$.

PRoposition 2. $e_{a}$ converges weakly to 0 in $\mathscr{D}$ as $|a| \rightarrow 1$.

PROOF. Let $f \in \mathscr{D}$ be an arbitrary function. Since $e_{a}(0)=0$ and $\left\langle\mathcal{R} f, B_{a}\right\rangle_{2}=\mathcal{R} f(a)$, we see

$$
\left\langle f, e_{a}\right\rangle=\frac{1}{\left\|E_{a}\right\|}\left\langle\mathcal{R} f, B_{a}-1\right\rangle_{2}=\frac{1}{\left\|E_{a}\right\|}[\mathcal{R} f(a)-\mathcal{R} f(0)]
$$

for all $a \in B$. Recall that

$$
\lim _{|z| \rightarrow 1}\left(1-|z|^{2}\right)^{\frac{n+1}{2}}|F(z)|=0
$$

for every $F \in A^{2}$; see [14, Theorem 2.1] and its remark. It follows that

$$
\lim _{|a| \rightarrow 1}\left\langle f, e_{a}\right\rangle=\lim _{|a| \rightarrow 1} \frac{\left(1-|a|^{2}\right)^{\frac{n+1}{2}}}{\sqrt{1-\left(1-|a|^{2}\right)^{n+1}}}[\mathcal{R} f(a)-\mathcal{R} f(0)]=0,
$$

so $e_{a}$ converges to 0 weakly in $\mathscr{D}$ as $|a| \rightarrow 1$. The proof is complete.

By (6), we see that the identity operator from $\mathscr{D}$ into $A^{2}$ is bounded. Moreover, it is compact as shown in the following lemma.

LEMMA 3. The identity operator from $\mathscr{D}$ into $A^{2}$ is compact. 
Proof. Using (6), we see that any bounded sequence in $\mathscr{D}$-norm is bounded in $A^{2}$ norm. So, it is locally bounded and then constitutes a normal family. Hence the sequence has a subsequence converging locally uniformly in $B$ to a function in $A^{2}$. So, the identity operator is compact, as desired. The proof is complete.

As an application of Lemma 3 , we remark in passing that for any sequence $\left\{f_{j}\right\}$ converging weakly to 0 in $\mathscr{D}$, the sequence $\left\|f_{j}\right\|_{2}$ converges to 0 as $j \rightarrow \infty$.

Recall that $Q$ is the Bergman projection. The following lemma will be useful in our proofs.

LEMMA 4. Let $u \in \mathscr{L}^{1, \infty}$. If a sequence $f_{j}$ converges to 0 weakly in $\mathscr{D}$, then we have

$$
\lim _{j \rightarrow \infty} Q\left[\mathcal{R}\left(u f_{j}\right)\right](0)=0 .
$$

ProOF. We first claim that $\mathcal{R} f_{j}$ converges to 0 weakly in $A^{2}$. To prove this, let $\varphi \in A^{2}$ be an arbitrary function and choose $\psi \in \mathscr{D}$ such that $\mathcal{R} \psi=\varphi-\varphi(0)$. Since $\mathcal{R} f_{j}(0)=0$ for each $j$, we see

$$
\begin{aligned}
\left\langle\mathcal{R} f_{j}, \varphi\right\rangle_{2} & =\left\langle\mathcal{R} f_{j}, \mathcal{R} \psi+\varphi(0)\right\rangle_{2} \\
& =\left\langle\mathcal{R} f_{j}, \mathcal{R} \psi\right\rangle_{2}+\overline{\varphi(0)} \mathcal{R} f_{j}(0) \\
& =\left\langle\mathcal{R} f_{j}, \mathcal{R} \psi\right\rangle_{2} \\
& =\left\langle f_{j}, \psi\right\rangle-f_{j}(0) \overline{\psi(0)}
\end{aligned}
$$

for each $j$. On the other hand, by the remark just after Lemma 3, we have

$$
\left|f_{j}(0)\right|=\left|\int_{B} f_{j} d V\right| \leq\left(\int_{B}\left|f_{j}\right|^{2} d V\right)^{\frac{1}{2}} \rightarrow 0, \quad j \rightarrow \infty .
$$

Since $f_{j}$ converges to 0 weakly by assumption, we see $\left\langle\mathcal{R} f_{j}, \varphi\right\rangle_{2} \rightarrow 0$ as $j \rightarrow \infty$ and $\mathcal{R} f_{j}$ converges to 0 weakly in $A^{2}$. To complete the proof, we note that

$$
\left|\int_{B}(\mathcal{R} u) f_{j} d V\right| \leq\|\mathcal{R} u\|_{\infty}\left(\int_{B}\left|f_{j}\right|^{2} d V\right)^{\frac{1}{2}} \rightarrow 0
$$

as $j \rightarrow \infty$ by the remark after Lemma 3 again. It follows that

$$
\begin{aligned}
\lim _{j \rightarrow \infty} Q\left[\mathcal{R}\left(u f_{j}\right)\right](0) & =\lim _{j \rightarrow \infty} \int_{B} \mathcal{R}\left(u f_{j}\right) d V \\
& =\lim _{j \rightarrow \infty} \int_{B}\left[(\mathcal{R} u) f_{j}+u\left(\mathcal{R} f_{j}\right)\right] d V \\
& =\lim _{j \rightarrow \infty}\left\langle\mathcal{R} f_{j}, \bar{u}\right\rangle_{2} \\
& =\lim _{j \rightarrow \infty}\left\langle\mathcal{R} f_{j}, Q \bar{u}\right\rangle_{2} \\
& =0
\end{aligned}
$$

because $\mathcal{R} f_{j}$ converges weakly to 0 in $A^{2}$. The proof is complete. 
In our characterization of the compactness for sums of products of two Toeplitz operators, we will use some known results on the Toeplitz operators on the Bergman space. Thus, we need to introduce the notion of Bergman space Toeplitz operators.

For a function $\varphi \in L^{\infty}$, we let $S_{\varphi}$ denote the Bergman space Toeplitz operator on $A^{2}$ defined by

$$
S_{\varphi} f=Q(\varphi f)
$$

for functions $f \in A^{2}$. Clearly $S_{\varphi}$ is a bounded linear operator on $A^{2}$.

Given a bounded linear operator $L$ on $A^{2}$, the Berezin transform $\widehat{L}$ of $L$ is the function on $B$ defined by

$$
\widehat{L}(a)=\int_{B}\left(L b_{a}\right) \overline{b_{a}} d V, \quad a \in B
$$

where $b_{a}$ denotes the normalized Bergman kernel of $A^{2}$ given by

$$
b_{a}(z)=\frac{1}{\left\|B_{a}\right\|_{2}} B_{a}(z), \quad z \in B .
$$

See Chapter 2 of [14] for details and related facts.

It is known that $\widehat{L}$ is a continuous function on $B$. Moreover, it turns out that the Berezin transform of an operator which is a product of Bergman space Toeplitz operators preserves the boundary continuity of symbols. More explicitly, it is known that for given symbols $\varphi, \psi \in$ $L^{\infty}$ which are continuous on $\bar{B}$, the Berezin transform $\widehat{S_{\varphi} S_{\psi}}$ is continuous up to $\bar{B}$ and

$$
\widehat{S_{\varphi} S_{\psi}}=\varphi \psi \quad \text { on } \quad \partial B
$$

holds; see Proposition 2.1 of [3] for example.

Also, the Berezin transform turns out to provide a compactness criterion for operators which are sums of products of Bergman space Toeplitz operators. Specially, for symbols $\varphi_{j}, \psi_{j} \in L^{\infty}$, it is known that $\sum_{j=1}^{N} S_{\varphi_{j}} S_{\psi_{j}}$ is compact on $A^{2}$ if and only if

$$
\lim _{|a| \rightarrow 1}\left[\sum_{j=1}^{N} \widehat{S_{\varphi_{j}} S_{\psi_{j}}}\right](a)=0 ;
$$

see Theorem A of [8] for more general results. Recall that each function in $\mathscr{L}^{1, \infty}$ can be extended to a continuous function on $\bar{B}$. Now, combining these observations with (10), we have the following characterization.

Lemma 5. Let $\varphi_{j}, \psi_{j} \in \mathscr{L}^{1, \infty}$ for $j=1, \ldots, N$. Then $\sum_{j=1}^{N} S_{\varphi_{j}} S_{\psi_{j}}$ is compact on $A^{2}$ if and only if $\sum_{j=1}^{N} \varphi_{j} \psi_{j}=0$ on $\partial B$.

Before we characterize compact sums of Toeplitz products, we first prove the following lemma which will be useful. For $a \in B$, we recall

$$
e_{a}(z)=\frac{1}{\left\|E_{a}\right\|} E_{a}(z), \quad z \in B
$$


Lemma 6. Let $u, v \in \mathscr{L}^{1, \infty}$ and $\zeta \in \partial B$. Then

$$
\lim _{a \rightarrow \zeta}\left\langle T_{u} T_{v} e_{a}, e_{a}\right\rangle=u(\zeta) v(\zeta)
$$

PROOF. Since $e_{a}(0)=0$ and $\mathcal{R} E_{a}=B_{a}-1$ for all $a \in B$, we first note that

$$
\begin{aligned}
\left\langle T_{u} T_{v} e_{a}, e_{a}\right\rangle & =\left\langle u T_{v} e_{a}, e_{a}\right\rangle \\
& =\left\langle(\mathcal{R} u) T_{v} e_{a}, \mathcal{R} e_{a}\right\rangle_{2}+\frac{1}{\left\|E_{a}\right\|^{2}}\left\langle u \mathcal{R}\left(T_{v} E_{a}\right), B_{a}-1\right\rangle_{2}
\end{aligned}
$$

for all $a \in B$. On the other hand, by (5), we have

$$
\begin{aligned}
\mathcal{R}\left(T_{v} E_{a}\right) & =\mathcal{R} P\left(v E_{a}\right) \\
& =Q\left[(\mathcal{R} v) E_{a}+v\left(B_{a}-1\right)\right]-Q\left[\mathcal{R}\left(v E_{a}\right)\right](0) \\
& =S_{\mathcal{R} v} E_{a}+S_{v} B_{a}-S_{v} 1-Q\left[\mathcal{R}\left(v E_{a}\right)\right](0)
\end{aligned}
$$

and hence

$$
\begin{aligned}
\left\langle u \mathcal{R}\left(T_{v} E_{a}\right), B_{a}-1\right\rangle_{2}= & \left\langle u \mathcal{R}\left(T_{v} E_{a}\right), B_{a}\right\rangle_{2}-\left\langle\mathcal{R}\left(T_{v} E_{a}\right), \bar{u}\right\rangle_{2} \\
= & \left\langle S_{u} S_{\mathcal{R} v} E_{a}, B_{a}\right\rangle_{2}+\left\langle S_{u} S_{v} B_{a}, B_{a}\right\rangle_{2}-\left\langle S_{u} S_{v} 1, B_{a}\right\rangle_{2} \\
& -Q\left[\mathcal{R}\left(v E_{a}\right)\right](0)\left\langle u, B_{a}\right\rangle_{2}-\left\langle\mathcal{R} T_{v} E_{a}, \bar{u}\right\rangle_{2}
\end{aligned}
$$

for all $a \in B$. Since $e_{a}$ converges weakly to 0 in $\mathscr{D}$ by Proposition 2, we see $T_{v} e_{a}$ also converges weakly to 0 as $|a| \rightarrow 1$ in $\mathscr{D}$. Also, note $\left\|\mathcal{R} e_{a}\right\|_{2}=\left\|e_{a}\right\|=1$ for all $a \in B$. It follows from Lemma 3 that

$$
\left|\left\langle(\mathcal{R} u) T_{v} e_{a}, \mathcal{R} e_{a}\right\rangle_{2}\right| \leq\|\mathcal{R} u\|_{\infty}\left\|T_{v} e_{a}\right\|_{2}\left\|\mathcal{R} e_{a}\right\|_{2}=\|\mathcal{R} u\|_{\infty}\left\|T_{v} e_{a}\right\|_{2} \rightarrow 0
$$

as $a \rightarrow \zeta$. Also, since $\left\|e_{a}\right\|_{2} \rightarrow 0$ as $a \rightarrow \zeta$ by Lemma 3 again, we see from (8)

$$
\begin{aligned}
\frac{1}{\left\|E_{a}\right\|^{2}}\left|\left\langle S_{u} S_{\mathcal{R} v} E_{a}, B_{a}\right\rangle_{2}\right| & =\frac{1}{\left\|E_{a}\right\|}\left|\left\langle S_{u} S_{\mathcal{R} v} e_{a}, B_{a}\right\rangle_{2}\right| \\
& \leq\left\|S_{u} S_{\mathcal{R} v} \mid\right\|\left\|e_{a}\right\|_{2} \frac{\left\|B_{a}\right\|_{2}}{\left\|E_{a}\right\|} \\
& \rightarrow 0, \quad a \rightarrow \zeta
\end{aligned}
$$

where $\|L\|$ denotes the operator norm of a bounded operator $L$. Also, we note

$$
\frac{1}{\left\|E_{a}\right\|^{2}}\left\langle S_{u} S_{v} B_{a}, B_{a}\right\rangle_{2}=\left(\frac{\left\|B_{a}\right\|_{2}}{\left\|E_{a}\right\|}\right)^{2}\left\langle S_{u} S_{v} b_{a}, b_{a}\right\rangle_{2}=\left(\frac{\left\|B_{a}\right\|_{2}}{\left\|E_{a}\right\|}\right)^{2} \widehat{S_{u} S_{v}}(a)
$$

for all $a \in B$ and by (8)

$$
\frac{1}{\left\|E_{a}\right\|^{2}}\left|\left\langle S_{u} S_{v} 1, B_{a}\right\rangle_{2}\right| \leq \sqrt{\frac{\left(1-|a|^{2}\right)^{n+1}}{1-\left(1-|a|^{2}\right)^{n+1}}}|| S_{u} S_{v} \| \frac{\left\|B_{a}\right\|_{2}}{\left\|E_{a}\right\|} \rightarrow 0
$$


as $a \rightarrow \zeta$. Similarly, we see by Lemma 4 and (8)

$$
\begin{aligned}
\frac{1}{\left\|E_{a}\right\|^{2}}\left|Q\left[\mathcal{R}\left(v E_{a}\right)\right](0)\left\langle u, B_{a}\right\rangle_{2}\right| & =\frac{1}{\left\|E_{a}\right\|}\left|Q\left[\mathcal{R}\left(v e_{a}\right)\right](0)\left\langle u, B_{a}\right\rangle_{2}\right| \\
& \leq \mid Q\left[\mathcal{R}\left(v e_{a}\right)\right](0)\|\| u \|_{\infty} \frac{\left\|B_{a}\right\|_{2}}{\left\|E_{a}\right\|} \\
& \rightarrow 0
\end{aligned}
$$

and

$$
\begin{aligned}
\frac{1}{\left\|E_{a}\right\|^{2}}\left|\left\langle\mathcal{R} T_{v} E_{a}, \bar{u}\right\rangle_{2}\right| & \leq \frac{1}{\left\|E_{a}\right\|}\left\|\mathcal{R} T_{v} e_{a}\right\|_{2}\|u\|_{2} \\
& \leq \frac{1}{\left\|E_{a}\right\|}\left\|T_{v} e_{a}\right\|\|u\|_{\infty} \\
& \leq \frac{1}{\left\|E_{a}\right\|}\left\|T_{v}\right\|\|u\|_{\infty} \\
& \rightarrow 0
\end{aligned}
$$

as $a \rightarrow \zeta$. It follows from (12) and (8) that

$$
\lim _{a \rightarrow \zeta} \frac{1}{\left\|E_{a}\right\|^{2}}\left\langle u \mathcal{R}\left(T_{v} E_{a}\right), B_{a}-1\right\rangle_{2}=\lim _{a \rightarrow \zeta} \widehat{S_{u} S_{v}}(a)
$$

Now, combining the above with (11) and (13), we see

$$
\lim _{a \rightarrow \zeta}\left\langle T_{u} T_{v} e_{a}, e_{a}\right\rangle=\lim _{a \rightarrow \zeta} \widehat{S_{u} S_{v}}(a)=u(\zeta) v(\zeta)
$$

by (10) because $u, v$ are continuous on $\bar{B}$. The proof is complete.

Now, we characterize the compactness for operators which are finite sums of products of two Toeplitz operators.

THEOREM 7. Let $u_{j}, v_{j} \in \mathscr{L}^{1, \infty}$ for $j=1, \ldots, N$. Then the following statements are equivalent.
(a) $\sum_{j=1}^{N} T_{u_{j}} T_{v_{j}}$ is compact on $\mathscr{D}$.
(b) $\sum_{j=1}^{N} u_{j} v_{j}=0$ on $\partial B$.

PROOF. First assume (a) and let $\zeta \in \partial B$ be arbitrary. Since $e_{a}$ converges weakly to 0 in $\mathscr{D}$ as $a \rightarrow \zeta$, we see by Lemma 6 ,

$$
0=\lim _{a \rightarrow \zeta}\left\langle\sum_{j=1}^{N} T_{u_{j}} T_{v_{j}} e_{a}, e_{a}\right\rangle=\sum_{j=1}^{N} \lim _{a \rightarrow \zeta}\left\langle T_{u_{j}} T_{v_{j}} e_{a}, e_{a}\right\rangle=\sum_{j=1}^{N} u_{j}(\zeta) v_{j}(\zeta)
$$

and (b) follows. 
Now, assume (b) and put

$$
T=\sum_{j=1}^{N} T_{u_{j}} T_{v_{j}}, \quad S=\sum_{j=1}^{N} S_{u_{j}} S_{v_{j}}
$$

for simplicity. Fixing $j$, we first note that

$$
\left|T_{u_{j}} T_{v_{j}} f(0)\right|=\left|P\left[u_{j} T_{v_{j}} f\right](0)\right| \leq \int_{B}\left|u_{j} T_{v_{j}} f\right| d V \leq\left\|u_{j}\right\|_{\infty}\left\|T_{v_{j}} f\right\|_{2}
$$

and then

$$
|T f(0)| \leq \sum_{j=1}^{N}\left\|u_{j}\right\|_{\infty}\left\|T_{v_{j}} f\right\|_{2}
$$

for every $f \in \mathscr{D}$. Also, by (5)

$$
\begin{aligned}
\mathcal{R}\left[T_{u_{j}} T_{v_{j}} f\right]= & \mathcal{R}\left[P\left(u_{j} P\left(v_{j} f\right)\right)\right] \\
= & Q\left[\mathcal{R}\left(u_{j} P\left(v_{j} f\right)\right)\right]-Q\left[\mathcal{R}\left(u_{j} P\left(v_{j} f\right)\right)\right](0) \\
= & Q\left[\left(\mathcal{R} u_{j}\right) T_{v_{j}} f\right]+Q\left[u_{j} \mathcal{R} P\left(v_{j} f\right)\right]-Q\left[\mathcal{R}\left(u_{j} P\left(v_{j} f\right)\right)\right](0) \\
= & Q\left[\left(\mathcal{R} u_{j}\right) T_{v_{j}} f\right]+Q\left[u_{j} Q\left(f \mathcal{R} v_{j}\right)\right]+Q\left[u_{j} Q\left(v_{j} \mathcal{R} f\right)\right] \\
& -Q\left(u_{j}\right) Q\left(\mathcal{R}\left(v_{j} f\right)\right)(0)-Q\left[\mathcal{R}\left(u_{j} P\left(v_{j} f\right)\right)\right](0) \\
= & S_{\mathcal{R} u_{j}} T_{v_{j}} f+S_{u_{j}} S_{\mathcal{R} v_{j}} f+S_{u_{j}} S_{v_{j}}(\mathcal{R} f) \\
& -Q\left(u_{j}\right) Q\left(\mathcal{R}\left(v_{j} f\right)\right)(0)-Q\left[\mathcal{R}\left(u_{j} P\left(v_{j} f\right)\right)\right](0)
\end{aligned}
$$

for every $f \in \mathscr{D}$. Thus,

$$
\begin{aligned}
\mathcal{R}(T f)= & \sum_{j=1}^{N} S_{\mathcal{R} u_{j}} T_{v_{j}} f+\sum_{j=1}^{N} S_{u_{j}} S_{\mathcal{R} v_{j}} f+S(\mathcal{R} f) \\
& -\sum_{j=1}^{N} Q\left(u_{j}\right) Q\left(\mathcal{R}\left(v_{j} f\right)\right)(0)-\sum_{j=1}^{N} Q\left[\mathcal{R}\left(u_{j} P\left(v_{j} f\right)\right)\right](0)
\end{aligned}
$$

for every $f \in \mathscr{D}$. Now, in order to prove (a), let $f_{k}$ be a sequence converging weakly to 0 in $\mathscr{D}$. Then, we need to show that $\left\|T f_{k}\right\| \rightarrow 0$ as $k \rightarrow \infty$. For each $j$, since $T_{v_{j}} f_{k}$ also converges weakly to 0 in $\mathscr{D}$, we note that $\left\|f_{k}\right\|_{2}$ and $\left\|T_{v_{j}} f_{k}\right\|_{2}$ converge to 0 as $k \rightarrow \infty$ by Lemma 3. Thus, by (14), we see

$$
\lim _{k \rightarrow \infty}\left|T f_{k}(0)\right| \leq \lim _{k \rightarrow \infty} \sum_{j=1}^{N}\left\|u_{j}\right\|_{\infty}\left\|T_{v_{j}} f_{k}\right\|_{2}=0 .
$$

Also, by Lemma 4, we see $Q\left(\mathcal{R}\left(v_{j} f_{k}\right)\right)(0)$ and $Q\left[\mathcal{R}\left(u_{j} T_{v_{j}} f_{k}\right)\right](0)$ converge to 0 as $k \rightarrow \infty$ for each $j$. Also, since $\mathcal{R} f_{k}$ converges weakly to 0 in $A^{2}$ and $S$ is compact by assumption (b) 
and Lemma 5, we see $\left\|S\left(\mathcal{R} f_{k}\right)\right\|_{2} \rightarrow 0$ as $k \rightarrow \infty$. It follows from (15) that

$$
\begin{aligned}
\left\|\mathcal{R}\left(T f_{k}\right)\right\|_{2} & \leq \sum_{j=1}^{N}\left\|S_{\mathcal{R} u_{j}}\right\|\left\|T_{v_{j}} f_{k}\right\|_{2}+\left\|f_{k}\right\|_{2} \sum_{j=1}^{N}\left\|S_{u_{j}} S_{\mathcal{R} v_{j}}\right\|+\left\|S\left(\mathcal{R} f_{k}\right)\right\|_{2} \\
& \left.+\sum_{j=1}^{N}\left\|Q\left(u_{j}\right)\right\|_{2}\left|Q\left(\mathcal{R}\left(v_{j} f_{k}\right)\right)(0)\right|+\sum_{j=1}^{N} \mid Q\left[\mathcal{R}\left(u_{j} T_{v_{j}} f_{k}\right)\right)\right](0) \mid,
\end{aligned}
$$

which converges to 0 as $k \rightarrow \infty$. Now, combining this observation with (16), we see

$$
\lim _{k \rightarrow \infty}\left\|T f_{k}\right\|^{2}=\lim _{k \rightarrow \infty}\left(\left|T f_{k}(0)\right|^{2}+\left\|\mathcal{R}\left(T f_{k}\right)\right\|_{2}^{2}\right)=0
$$

and hence $T$ is compact on $\mathscr{D}$ as desired. The proof is complete.

As a simple application of Theorem 7 , we have the following corollary which will be used in the description of the Toeplitz algebra $\mathcal{T}$ in Section 4.

COROLlary 8. For $u, v \in \mathscr{L}^{1, \infty}$, the semi-commutator $T_{u} T_{v}-T_{u v}$ and commutator $T_{u} T_{v}-T_{v} T_{u}$ are always compact on $\mathscr{D}$.

Since the Toeplitz operator with symbol 1 is the identity operator, we have the following characterization of compact Toepltz operators.

COROllary 9. For $u \in \mathscr{L}^{1, \infty}, T_{u}$ is compact on $\mathscr{D}$ if and only if $u=0$ on $\partial B$.

On the smaller Dirichlat space $\mathscr{D}_{0}$ of the unit disk $B_{1}$, it is known that for a Toeplitz operator with symbol $u$, it is compact on $\mathscr{D}_{0}$ if and only if it equals 0 on $\mathscr{D}_{0}$ if and only if $u=0$ on $\partial B_{1}$; see Proposition 3.1 and Theorem 3.4 of [12]. In view of Corollary 9, one might ask whether the same is true on our Dirichlet space $\mathscr{D}$. But the answer is no. For example, the Toeplitz operator $T_{1-|z|^{2}}$ is compact on $\mathscr{D}$ by Theorem 7, but it is easy to see that $T_{1-|z|^{2}}$ is not equal to 0 . Indeed,

$$
T_{1-|z|^{2}} 1=\int_{B}\left(1-|w|^{2}\right) d V(w) \neq 0 .
$$

This fact will be also used in the description of the Toeplitz algebra $\mathcal{T}$.

As another application of Theorem 7, we consider the compact product problem of when compactness of a product of two Toeplitz operators on $\mathscr{D}$ implies the triviality of one of symbols. The example $T_{1} T_{1-|z|^{2}}=T_{1-|z|^{2}}$ shows that the compact product problem fails for general symbols. But our result below shows that the problem can be solvable for pluriharmonic symbols. Recall that a twice continuously differentiable function $u$ on $B$ is said to be pluriharmonic if

$$
\frac{\partial^{2} u}{\partial z_{i} \bar{\partial} z_{j}}=0, \quad i, j=1, \ldots, n
$$

on $B$. For $n \geq 2$ and two pluriharmonic functions $u, v$ which are continuous on $\partial B$, it is known that $u v=0$ on $\partial B$ if and only if either $u=0$ or $v=0$; see Corollary 3.5 of [4] for more general result. Thus, the following is a simple consequence of Theorem 7 . 
COROLlaRy $10(n \geq 2)$. Let $u, v \in \mathscr{L}^{1, \infty}$ be pluriharmonic functions. Then $T_{u} T_{v}$ is compact on $\mathscr{D}$ if and only if either $u=0$ or $v=0$.

We also remark in passing that Corollary 10 can not be extended to the one dimensional case in general. To see an example, consider two harmonic symbols $u, v$ which are nonzero on the unit disk $B_{1}$ and $u v=0$ on $\partial B_{1}$. Then $T_{u} T_{v}$ is compact by Theorem 7 , but neither $u$ nor $v$ is identically zero.

But, for a single Toeplitz operator with pluriharmonic symbol, we have the following characterization for full range of dimensions.

COROLlary 11. Let $u \in \mathscr{L}^{1, \infty}$ be a pluriharmonic function. Then the following are equivalent.

(a) $T_{u}$ is compact on $\mathscr{D}$.

(b) $u=0$ on $B$.

(c) $T_{u}=0$ on $\mathscr{D}$.

PROOF. Since a pluriharmonic symbol which vanishes on $\partial B$ vanishes on $B$, implication (a) $\Rightarrow$ (b) follows from Theorem 7. Also, since (b) $\Rightarrow$ (c) $\Rightarrow$ (a) is clear, we complete the proof.

4. Toeplitz algebra. In this section, we characterize Fredholm Toeplitz operators and describe the essential norm of a Toeplitz operator. Also, we establish a short exact sequence associated with the Toeplitz algebra $\mathcal{T}$.

We let $\mathcal{B}$ denote the $C^{*}$-algebra consisting of all bounded operators on $\mathscr{D}$. Also, let $\mathcal{K}$ be the algebra of all compact operators on $\mathscr{D}$. An operator $L \in \mathcal{B}$ is said to be Fredholm if $L+\mathcal{K}$ is invertible in the quotient algebra $\mathcal{B} / \mathcal{K}$. Recall that $L \in \mathcal{B}$ is Fredholm if and only if there exist $L_{1}, L_{2} \in \mathcal{B}$ such that $L_{1} L-I, L L_{2}-I \in \mathcal{K}$. Also, if there exists a sequence $\left\{f_{j}\right\}$ of unit vectors in $\mathscr{D}$ for which $f_{j} \rightarrow 0$ weakly and $\left\|L f_{j}\right\| \rightarrow 0$ as $j \rightarrow \infty$, then $L$ can't be Fredholm; see Chapter 6 of [6] for example.

We characterize Fredholm Toeplitz operators.

THEOREM 12. Let $u \in \mathscr{L}^{1, \infty}$. Then $T_{u}$ is Fredholm on $\mathscr{D}$ if and only if $u$ has no zero on $\partial B$.

ProOF. First suppose $T_{u}$ is Fredholm on $\mathscr{D}$ and assume $u(\zeta)=0$ for some $\zeta \in \partial B$. Note that

$$
\left\|u e_{a}\right\|^{2}=\left|\int_{B} u e_{a} d V\right|^{2}+\left\langle\widetilde{\mathcal{R}}\left(u e_{a}\right), \widetilde{\mathcal{R}}\left(u e_{a}\right)\right\rangle_{2}+\left\langle\mathcal{R}\left(u e_{a}\right), \mathcal{R}\left(u e_{a}\right)\right\rangle_{2}
$$

for all $a \in B$. Since $e_{a}$ converges weakly to 0 in $\mathscr{D}$ as $a \rightarrow \zeta$ by Proposition 2, we note $\left\|e_{a}\right\|_{2} \rightarrow 0$ as $a \rightarrow \zeta$ by Lemma 3. Thus, we have

$$
\lim _{a \rightarrow \zeta}\left|\int_{B} u e_{a} d V\right|^{2} \leq \lim _{a \rightarrow \zeta}\|u\|_{\infty}^{2} \int_{B}\left|e_{a}\right|^{2} d V=0
$$


and similarly

$$
\left|\left\langle\widetilde{\mathcal{R}}\left(u e_{a}\right), \widetilde{\mathcal{R}}\left(u e_{a}\right)\right\rangle_{2}\right|=\left|\left\langle e_{a} \widetilde{\mathcal{R}} u, e_{a} \widetilde{\mathcal{R}} u\right\rangle_{2}\right| \leq\|\widetilde{\mathcal{R}} u\|_{\infty}^{2} \int_{B}\left|e_{a}\right|^{2} d V \rightarrow 0
$$

as $a \rightarrow \zeta$. Now, to estimate $\left\langle\mathcal{R}\left(u e_{a}\right), \mathcal{R}\left(u e_{a}\right)\right\rangle_{2}$, we first note

$$
\left|\left\langle e_{a} \mathcal{R} u, e_{a} \mathcal{R} u\right\rangle_{2}\right| \leq\|\mathcal{R} u\|_{\infty}^{2}\left\|e_{a}\right\|_{2}^{2}
$$

and

$$
\begin{aligned}
\left|\left\langle e_{a} \mathcal{R} u, u \mathcal{R}\left(e_{a}\right)\right\rangle_{2}\right| & \leq \frac{1}{\left\|E_{a}\right\|} \int_{B}\left|e_{a}(\mathcal{R} u) \overline{u\left(B_{a}-1\right)}\right| d V \\
& \leq\|u \mathcal{R} u\|_{\infty}\left\|e_{a}\right\|_{2} \frac{\left\|B_{a}\right\|_{2}+1}{\left\|E_{a}\right\|}
\end{aligned}
$$

for all $a \in B$. Also, by a simple calculation, we see

$$
\begin{aligned}
\left|\left\langle u \mathcal{R}\left(e_{a}\right), u \mathcal{R}\left(e_{a}\right)\right\rangle_{2}\right| & =\frac{1}{\left\|E_{a}\right\|^{2}}\left\langle u\left(B_{a}-1\right), u\left(B_{a}-1\right)\right\rangle_{2} \\
& \leq \frac{1}{\left\|E_{a}\right\|^{2}}\left(\left\langle u B_{a}, u B_{a}\right\rangle_{2}+2\left|\left\langle u B_{a}, u\right\rangle_{2}\right|+\left|\langle u, u\rangle_{2}\right|\right) \\
& \leq\left(\frac{\left\|B_{a}\right\|_{2}}{\| E_{a}||}\right)^{2}\left\langle S_{|u|^{2}} b_{a}, b_{a}\right\rangle_{2}+\frac{2\|u\|_{\infty}^{2}\left\|B_{a}\right\|_{2}+\|u\|_{\infty}^{2}}{\left\|E_{a}\right\|^{2}} \\
& \leq\left(\frac{\left\|B_{a}\right\|_{2}}{\| E_{a}||}\right)^{2} \widehat{S_{|u|^{2}}}(a)+\frac{3\|u\|_{\infty}^{2}\left\|B_{a}\right\|_{2}}{\left\|E_{a}\right\|^{2}}
\end{aligned}
$$

for all $a \in B$. Since $\left\|e_{a}\right\|_{2} \rightarrow 0$ as $|a| \rightarrow 1$ and

$$
\begin{aligned}
\left\langle\mathcal{R}\left(u e_{a}\right), \mathcal{R}\left(u e_{a}\right)\right\rangle_{2}= & \left\langle e_{a} \mathcal{R} u, e_{a} \mathcal{R} u\right\rangle_{2}+\left\langle e_{a} \mathcal{R} u, u \mathcal{R}\left(e_{a}\right)\right\rangle_{2} \\
& +\left\langle u \mathcal{R}\left(e_{a}\right), e_{a} \mathcal{R} u\right\rangle_{2}+\left\langle u \mathcal{R}\left(e_{a}\right), u \mathcal{R}\left(e_{a}\right)\right\rangle_{2}
\end{aligned}
$$

for all $a \in B$, the above observations together with (8) show

$$
\lim _{a \rightarrow \zeta}\left\langle\mathcal{R}\left(u e_{a}\right), \mathcal{R}\left(u e_{a}\right)\right\rangle_{2} \leq \lim _{a \rightarrow \zeta} \widehat{S_{|u|^{2}}}(a)=|u(\zeta)|^{2}
$$

by (10) because $|u|^{2}$ is continuous on $\bar{B}$. Now, combining the above with (17), (18) and (19), we see

$$
\lim _{a \rightarrow \zeta}\left\|T_{u} e_{a}\right\|^{2}=\lim _{a \rightarrow \zeta}\left\|P\left(u e_{a}\right)\right\|^{2} \leq \lim _{a \rightarrow \zeta}\left\|u e_{a}\right\|^{2} \leq|u(\zeta)|^{2}=0
$$

because $u(\zeta)=0$. Since the sequence $\left\{e_{a}\right\}$ of unit vectors converges weakly to 0 in $\mathscr{D}, T_{u}$ can't be Fredholm on $\mathscr{D}$. Hence $u$ has no zero on $\partial B$.

Now, to prove the converse implication, assume $u$ has no zero on $\partial B$. Then $S_{u}$ is Fredholm on $A^{2}$ by Theorem 1.2 of [13]. Suppose $T_{u}$ is not Fredholm on $\mathscr{D}$. Thus, there is a sequence $\left\{k_{j}\right\}$ of unit vectors in $\mathscr{D}$ converging weakly to 0 for which $\left\|T_{u} k_{j}\right\| \rightarrow 0$ or $\left\|T_{u}^{*} k_{j}\right\| \rightarrow 0$ as $j \rightarrow \infty$; see [6, Chapter 6] for example. First suppose $\left\|T_{u} k_{j}\right\| \rightarrow 0$ as $j \rightarrow \infty$. Since $S_{u}$ is Fredholm on $A^{2}$, there exists a bounded operator $M$ on $A^{2}$ such that 
$M S_{u}-I$ is compact on $A^{2}$. Put $t_{j}=\mathcal{R} k_{j}$. Then we recall $t_{j} \rightarrow 0$ weakly on $A^{2}$ and note from (9)

$$
\lim _{j \rightarrow \infty}\left\|t_{j}\right\|_{2}^{2}=\lim _{j \rightarrow \infty}\left(\left\|k_{j}\right\|^{2}-\left|k_{j}(0)\right|^{2}\right)=\lim _{j \rightarrow \infty}\left\|k_{j}\right\|^{2}=1 .
$$

Since $M S_{u}-I$ is compact on $A^{2}$, we have $\left\langle\left(M S_{u}-I\right) t_{j}, t_{j}\right\rangle_{2} \rightarrow 0$ as $j \rightarrow \infty$ and hence

$$
\lim _{j \rightarrow \infty}\left\langle M S_{u} t_{j}, t_{j}\right\rangle_{2}=\lim _{j \rightarrow \infty}\left\|t_{j}\right\|_{2}^{2}=1 .
$$

Also, since $\left\|k_{j}\right\|_{2} \rightarrow 0$ as $j \rightarrow \infty$ by Lemma 3 , we have

$$
\left|\left\langle M Q(\mathcal{R} u) k_{j}, t_{j}\right\rangle_{2}\right| \leq\left\|M\left|\||| \mathcal{R} u\|_{\infty}\left\|k_{j}\right\|_{2}\right| \mid t_{j}\right\|_{2} \rightarrow 0
$$

as $j \rightarrow \infty$. It follows that

$$
\begin{aligned}
\left\langle M Q\left[\mathcal{R}\left(u k_{j}\right)\right], t_{j}\right\rangle_{2} & =\left\langle M Q\left[(\mathcal{R} u) k_{j}\right], t_{j}\right\rangle_{2}+\left\langle M Q\left(u t_{j}\right), t_{j}\right\rangle_{2} \\
& =\left\langle M Q(\mathcal{R} u) k_{j}, t_{j}\right\rangle_{2}+\left\langle M S_{u} t_{j}, t_{j}\right\rangle_{2} \\
& \rightarrow 1
\end{aligned}
$$

as $j \rightarrow \infty$. Also, note $Q\left[\mathcal{R}\left(u k_{j}\right)\right](0) \rightarrow 0$ by Lemma 4 and $\left\langle M 1, t_{j}\right\rangle_{2} \rightarrow 0$ because $t_{j} \rightarrow 0$ weakly on $A^{2}$ as $j \rightarrow \infty$. It follows from (5) and (20) that

$$
\begin{aligned}
\left\langle M \mathcal{R}\left(T_{u} k_{j}\right), t_{j}\right\rangle_{2} & =\left\langle M \mathcal{R}\left[P\left(u k_{j}\right)\right], t_{j}\right\rangle_{2} \\
& =\left\langle M Q\left[\mathcal{R}\left(u k_{j}\right)\right], t_{j}\right\rangle_{2}-Q\left[\mathcal{R}\left(u k_{j}\right)\right](0)\left\langle M 1, t_{j}\right\rangle_{2} \\
& \rightarrow 1
\end{aligned}
$$

as $j \rightarrow \infty$. On the other hand, since $\left\|T_{u} k_{j}\right\| \rightarrow 0$ and $\left\|t_{j}\right\|_{2} \rightarrow 1$, we see

$$
\left|\left\langle M \mathcal{R}\left(T_{u} k_{j}\right), t_{j}\right\rangle_{2}\right| \leq\left\|M \mathcal{R}\left(T_{u} k_{j}\right)\right\|_{2}\left\|t_{j}\right\|_{2} \leq\|M\||| T_{u} k_{j}\left|\left\|\mid t_{j}\right\|_{2} \rightarrow 0\right.
$$

as $j \rightarrow \infty$, which contradicts to (21).

Also, by the similar argument, we can see that the case $\left\|T_{u}^{*} k_{j}\right\| \rightarrow 0$ yields a contradiction. Hence $T_{u}$ is Fredholm on $\mathscr{D}$. The proof is complete.

Recall that the essential spectrum $\sigma_{e}(L)$ of $L \in \mathcal{B}$ is defined to be the spectrum of $L+\mathcal{K}$ in $\mathcal{B} / \mathcal{K}$. Thus the following is a simple consequence of Theorem 12.

Corollary 13. For $u \in \mathscr{L}^{1, \infty}$, we have $\sigma_{e}\left(T_{u}\right)=u(\partial B)$.

In conjunction with Corollary 9 , we compute the essential norm of a Toeplitz operator on $\mathscr{D}$. Given $L \in \mathcal{B}$, the essential norm $\|L\|_{e}$ of $L$ is defined as the norm of $L+\mathcal{K}$ in the quotient algebra $\mathcal{B} / \mathcal{K}$. That is,

$$
\|L\|_{e}=\inf _{K \in \mathcal{K}}\|L+K\| .
$$

The following gives a formula of the essential norm of a Toeplitz operator.

THEOREM 14. Given $u \in \mathscr{L}^{1, \infty}$, we have $\left\|T_{u}\right\|_{e}=\max _{\eta \in \partial B}|u(\eta)|$. 
Proof. Put $\rho=\max _{\eta \in \partial B}|u(\eta)|$ for simplicity. Choose a point $\zeta \in \partial B$ such that $|u(\zeta)|=\rho$. For any $K \in \mathcal{K}$, since $e_{a}$ converges weakly to 0 in $\mathscr{D}$ as $|a| \rightarrow 1$ by Proposition 2 , we note that

$$
\begin{aligned}
\left\|T_{u}+K\right\| & \geq \lim _{a \rightarrow \zeta}\left|\left\langle\left(T_{u}+K\right) e_{a}, e_{a}\right\rangle\right| \\
& =\lim _{a \rightarrow \zeta}\left|\left\langle T_{u} e_{a}, e_{a}\right\rangle\right| \\
& =|u(\zeta)|
\end{aligned}
$$

by Lemma 6 (with $v=1$ ), thus $\rho \leq\left\|T_{u}\right\|_{e}$ holds.

Now, we prove the reverse inequality. By Lemma 1.2 of [9], there is an orthonormal sequence $\left\{f_{j}\right\}$ in $\mathscr{D}$ for which $\left\|T_{u} f_{j}\right\| \rightarrow\left\|T_{u}\right\|_{e}$ as $j \rightarrow \infty$. In particular, since the sequence $\left\{f_{j}\right\}$ converges weakly to 0 in $\mathscr{D},\left\{f_{j}\right\}$ converges uniformly to 0 on every compact subsets of $B$. Let $\varepsilon>0$ be arbitrary. Using Lemma 3 , we can choose an integer $j_{1} \geq 0$ such that

$$
\int_{B}\left|f_{j}\right|^{2} d V<\varepsilon
$$

for every $j>j_{1}$. On the other hand, since $u$ is continuous on $\bar{B}$, there exists $r \in(0,1)$ such that $|u(z)| \leq \rho+\varepsilon$ for every $r<|z|<1$. Fix $t \in(r, 1)$. Then

$$
\lim _{j \rightarrow \infty} \int_{|z| \leq t}\left|f_{j}\right|^{2} d V=0 \text {. }
$$

Writing

$$
f_{j}(z)=\sum_{\alpha} a_{\alpha}^{j} z^{\alpha}
$$

for the Taylor series expansions of $f_{j}$, we note

$$
\int_{|z| \leq t}\left|f_{j}\right|^{2} d V=\sum_{|\alpha| \geq 0}\left|a_{\alpha}^{j}\right|^{2} \int_{|z| \leq t}\left|z^{\alpha}\right|^{2} d V=\sum_{|\alpha| \geq 0}\left|a_{\alpha}^{j}\right|^{2} \frac{t^{2|\alpha|+2 n}}{|\alpha|+n} \int_{\partial B}\left|\zeta^{\alpha}\right|^{2} d \sigma
$$

and

$$
\begin{aligned}
\int_{|z| \leq r}\left|\mathcal{R} f_{j}\right|^{2} d V & =\sum_{|\alpha|>0}\left|a_{\alpha}^{j}\right|^{2}|\alpha|^{2} \frac{r^{2|\alpha|+2 n}}{|\alpha|+n} \int_{\partial B}\left|\zeta^{\alpha}\right|^{2} d \sigma \\
& =\sum_{|\alpha|>0}\left|a_{\alpha}^{j}\right|^{2} \frac{t^{2|\alpha|+2 n}}{|\alpha|+n}|\alpha|^{2}\left(\frac{r}{t}\right)^{2|\alpha|+2 n} \int_{\partial B}\left|\zeta^{\alpha}\right|^{2} d \sigma
\end{aligned}
$$

for each $j$. Since $|\alpha|^{2}\left(\frac{r}{t}\right)^{2|\alpha|+2 n} \rightarrow 0$ as $|\alpha| \rightarrow \infty$, the observations above show that

$$
\lim _{j \rightarrow \infty} \int_{|z| \leq r}\left|\mathcal{R} f_{j}(z)\right|^{2} d V(z)=0 .
$$

Thus, there exists an integer $j_{2} \geq 0$ such that

$$
\int_{|z| \leq r}\left|\mathcal{R} f_{j}\right|^{2} d V<\varepsilon
$$


for every $j>j_{2}$. Since $\left\|f_{j}\right\|=1$ for each $j$, we have

$$
\begin{aligned}
\int_{B}\left|u \mathcal{R} f_{j}\right|^{2} d V & =\int_{|z| \leq r}\left|u \mathcal{R} f_{j}\right|^{2} d V+\int_{|z|>r}\left|u \mathcal{R} f_{j}\right|^{2} d V \\
& \leq\|u\|_{\infty}^{2} \int_{|z| \leq r}\left|\mathcal{R} f_{j}\right|^{2} d V+(\rho+\varepsilon)^{2} \int_{|z|>r}\left|\mathcal{R} f_{j}\right|^{2} d V \\
& \leq\|u\|_{\infty}^{2} \varepsilon+(\rho+\varepsilon)^{2}
\end{aligned}
$$

for every $j>j_{2}$. Thus, letting $C=\max \left\{\|u\|_{\infty},\|\mathcal{R} u\|_{\infty},\|\widetilde{\mathcal{R}} u\|_{\infty}\right\}$, we see

$$
\begin{aligned}
\left\|T_{u} f_{j}\right\| & \leq\left\|u f_{j}\right\| \\
& =\left[\left|\int_{B} u f_{j} d V\right|^{2}+\int_{B}\left(\left|(\mathcal{R} u) f_{j}+u \mathcal{R} f_{j}\right|^{2}+\left|(\widetilde{\mathcal{R}} u) f_{j}\right|^{2}\right) d V\right]^{\frac{1}{2}} \\
& \leq 2 C\left(\int_{B}\left|f_{j}\right|^{2} d V\right)^{\frac{1}{2}}+\left(\int_{B}\left|u \mathcal{R} f_{j}\right|^{2} d V\right)^{\frac{1}{2}} \\
& \leq 2 C \sqrt{\varepsilon}+\|u\|_{\infty} \sqrt{\varepsilon}+(\rho+\varepsilon)
\end{aligned}
$$

for every $j>\max \left\{j_{1}, j_{2}\right\}$ and $\varepsilon>0$, which means that $\lim _{j \rightarrow \infty}\left\|T_{u} f_{j}\right\| \leq \rho$. Now, recalling $\lim _{j \rightarrow \infty}\left\|T_{u} f_{j}\right\|=\left\|T_{u}\right\|_{e}$, we have $\left\|T_{u}\right\|_{e} \leq \rho$, as desired. The proof is complete.

On the Hardy space or Bergman space, it is known that the adjoint operator of a Toeplitz operator with symbol $u$ is another Toeplitz operator with symbol $\bar{u}$. We don't know whether the same is true on the Dirichlet space. But we show $T_{u}^{*}-T_{\bar{u}}$ is compact on $\mathscr{D}$ as shown in the following lemma which will be used in our description of Toeplitz algebra $\mathcal{T}$. The notation $T_{u}^{*}$ denotes the adjoint operator of $T_{u}$.

LEMMA 15. For $u \in \mathscr{L}^{1, \infty}, T_{u}^{*}-T_{\bar{u}}$ is compact on $\mathscr{D}$.

PROOF. First note that

$$
\left\langle\left(T_{u}^{*}-T_{\bar{u}}\right) f, g\right\rangle=f(0) \int_{B} \overline{u g} d V-\bar{g}(0) \int_{B} \bar{u} f d V+\langle\mathcal{R} f, g \mathcal{R} u\rangle_{2}-\langle f \mathcal{R} \bar{u}, \mathcal{R} g\rangle_{2}
$$

for every $f, g \in \mathscr{D}$. Also, note that

$$
\begin{gathered}
\left|f(0) \int_{B} \overline{u g} d V\right| \leq\|f\|_{2}\|u\|_{\infty}\|g\|_{2}, \\
\left|\bar{g}(0) \int_{B} \bar{u} f d V\right| \leq\|g\|_{2}\|u\|_{\infty}\|f\|_{2}
\end{gathered}
$$

and

$$
\begin{gathered}
\left|\langle\mathcal{R} f, g \mathcal{R} u\rangle_{2}\right| \leq\|\mathcal{R} f\|_{2}\|g\|_{2}\|\mathcal{R} u\|_{\infty}, \\
\left|\langle f \mathcal{R} \bar{u}, \mathcal{R} g\rangle_{2}\right| \leq\|f\|_{2}\|\mathcal{R} g\|_{2}\|\mathcal{R} \bar{u}\|_{\infty}
\end{gathered}
$$


for all $f, g \in \mathscr{D}$. Now, to prove the compactness of $T_{u}^{*}-T_{\bar{u}}$, let $f_{j}$ be any sequence converging weakly to 0 on $\mathscr{D}$. By the observations above with $f=f_{j}$ and $g=\left(T_{u}^{*}-T_{\bar{u}}\right) f_{j}$, we see

$$
\begin{aligned}
\left\|\left(T_{u}^{*}-T_{\bar{u}}\right) f_{j}\right\|^{2} \leq & 2\|u\|_{\infty}\left\|f_{j}\right\|_{2}\left\|g_{j}\right\|_{2} \\
& +\|\mathcal{R} u\|_{\infty}\left\|\mathcal{R} f_{j}\right\|_{2}\left\|g_{j}\right\|_{2}+\left\|f_{j}\right\|_{2}\|\mathcal{R} \bar{u}\|_{\infty}\left\|\mathcal{R} g_{j}\right\|_{2}
\end{aligned}
$$

where $g_{j}=\left(T_{u}^{*}-T_{\bar{u}}\right) f_{j}$. Note that $g_{j} \rightarrow 0$ weakly in $\mathscr{D}$ as $j \rightarrow \infty$. Hence, by Lemma 3, we see $\left\|f_{j}\right\|_{2}$ and $\left\|g_{j}\right\|_{2}$ converge to 0 as $j \rightarrow \infty$. Also, since a weakly convergent sequence is bounded in norm and $\left\|\mathcal{R} f_{j}\right\|_{2} \leq\left\|f_{j}\right\|$ for each $j$, we see $\left\|\mathcal{R} f_{j}\right\|_{2}$ is bounded. Also, $\left\|\mathcal{R} g_{j}\right\|_{2}$ is bounded by the same reason. Now, combining the observations above with (22), we see $\left\|\left(T_{u}^{*}-T_{\bar{u}}\right) f_{j}\right\| \rightarrow 0$ as $j \rightarrow \infty$ and hence $T_{u}^{*}-T_{\bar{u}}$ is compact on $\mathscr{D}$ as desired. The proof is complete.

Now, we establish a short exact sequence associated with Toeplitz algebra $\mathcal{T}$.

THEOREM 16. The following statements hold.

(a) The commutator ideal of $\mathcal{T}$ is the same as the ideal $\mathcal{K}$ of all compact operators on $\mathscr{D}$.

(b) The quotient algebra $\mathcal{T} / \mathcal{K}$ is $*$-isometrically isometric to $C(\partial B)$. That is, the sequence

$$
0 \rightarrow \mathcal{K} \rightarrow \mathcal{T} \rightarrow C(\partial B) \rightarrow 0
$$

is short exact.

Proof. We first show that the algebra $\mathcal{T}$ is irreducible. To show this, we will use the similar idea as in [5, Lemma 1] where the Bergman or Hardy space case was considered. Suppose $\mathcal{T}$ is reducible. Then there exists a nontrivial orthogonal projection $P_{0}$ for which $P_{0} T_{\varphi}=T_{\varphi} P_{0}$ for all $\varphi \in \mathscr{L}^{1, \infty}$. Put $f=P_{0} 1$. Then

$$
P_{0} \varphi=P_{0} T_{\varphi} 1=T_{\varphi} P_{0} 1=T_{\varphi} f=\varphi f
$$

for all holomorphic functions $\varphi \in \mathscr{L}^{1, \infty}$. In particular, we have $P_{0} K_{z}=K_{z} f$ for all $z \in B$. Putting

$$
k_{a}(z)=\frac{1}{\left\|K_{a}\right\|} K_{a}(z) \quad a, z \in B,
$$

we see from the reproducing property

$$
\begin{aligned}
P_{0} k_{a}(z) & =\left\langle P_{0} k_{a}, K_{z}\right\rangle=\left\langle k_{a}, P_{0} K_{z}\right\rangle=\frac{1}{\left\|K_{a}\right\|}\left\langle K_{a}, f K_{z}\right\rangle \\
& =\frac{1}{\left\|K_{a}\right\|} \overline{f(a) K_{z}(a)}=\frac{1}{\left\|K_{a}\right\|} \overline{f(a)} K_{a}(z)=\overline{f(a)} k_{a}(z)
\end{aligned}
$$

for all $a, z \in B$. Hence $\overline{f(a)}$ belongs to the spectrum of $P_{0}$ for all $a \in B$. Sine $P_{0}$ is a projection, its spectrum must be contained in $\{0,1\}$ and hence $f(B) \subset\{0,1\}$. Thus $f=0$ or $f=1$. Now, (23) shows that $P_{0} \varphi=T_{f} \varphi$ for all holomorphic polynomial $\varphi$. Note that the set of all holomorphic polynomials is dense in $\mathscr{D}$. Hence $P_{0}=T_{f}$ on $\mathscr{D}$. Now, since $f=0$ or $f=1$, we have $P_{0}=0$ or $P_{0}=I$, which is a contradiction because $P_{0}$ is nontrivial. Hence $\mathcal{T}$ is irreducible. 
Now, as mentioned at the remark just after Corollary 9, the Toeplitz operator $T_{1-|z|^{2}}$ is a nonzero compact operator on $\mathscr{D}$. So $\mathcal{T}$ contains a nonzero compact operator. By Theorem 5.39 of [7], we have $\mathcal{K} \subset \mathcal{T}$. Hence the commutator ideal of $\mathcal{T}$ contains the commutator ideal of $\mathcal{K}$, which is the same as $\mathcal{K}$. On the other hand, the commutator of two Toeplitz operators is compact by Corollary 8 , which implies that $\mathcal{K}$ contains the commutator ideal of $\mathcal{T}$, thus (a) holds.

Now, by noting $T_{u}^{*}-T_{\bar{u}} \in \mathcal{K}$ by Lemma 15 , one can see that $\left\{T_{u}+\mathcal{K}: u \in \mathscr{L}^{1, \infty}\right\}$ is a $C^{*}$-algebra. Define the symbol map $\xi:\left\{T_{u}+\mathcal{K}: u \in \mathscr{L}^{1, \infty}\right\} \rightarrow C(\partial B)$ by $\xi\left(T_{u}+\mathcal{K}\right)=u_{\mid \partial B}$. Then, by Corollary 8 and Theorem 14, we can see that $\xi$ is well-defined, one-to-one and an isometric $*$-homomorphism. Then, using the same argument as in Theorem 8 of [2], one can see that $\xi$ can be extended to $\mathcal{T} / \mathcal{K}$. Hence $\mathcal{T} / \mathcal{K}$ is *-isometrically isometric to $C(\partial B)$ and (b) holds. The proof is complete.

\section{REFERENCES}

[1] R. AdAms, Sobolev spaces, Pure and Applied Mathematics Vol. 65, Academic Press [A subsidiary of Harcourt Brace Jovanovich, Publishers], New York-London, 1975.

[ 2 ] G. CAO, Fredholm properties of Toeplitz operators on Dirichlet spaces, Pacific J. Math. 188 (1999), $209-223$.

[ 3 ] B. R. CHOE and H. Koo, Zero products of Toeplitz operators with harmonic symbols, J. Funct. Anal. 233 (2006), 307-334.

[ 4 ] B. R. ChOE, H. Koo and Y. J. LeE, Toeplitz products with pluriharmonic symbols on the Hardy space over the ball, J. Math. Anal. Appl. 381 (2011), 365-382.

[ 5 ] L. A. Coburn, Singular integral operators and Toeplitz operators on odd spheres, Indiana Univ. Math. J. 23 (1973), 433-439.

[6] J. B. Conway, A course in operator theory, Grad. Stud. Math. 21, American Mathematical Society, Providence, RI, 2000.

[ 7 ] R. G. Douglas, Banach algebra techniques in operator theory. Pure and Applied Mathematics, Vol. 49. Academic Press, New York-London, 1972.

[ 8 ] M. EnGLIš, Compact Toeplitz operators via the Berezin transform on bounded symmetric domains, Integral Equation and Operator Theory 33 (1999), 426-455.

[9] C. K. Fong, On the essential maximal numerical range, Acta Sci. Math. 41 (1979), 307-315.

[10] Y. J. LEE, Finite sums of Toeplitz products on the Dirichlet space, J. Math. Anal. Appl. 357 (2009), 504-515.

[11] Y. J. LEE and K. NA, Compact products of Toeplitz operators on the Dirichlet space of the unit ball, J. Math. Anal. Appl. 401 (2013), 654-658.

[12] Y. J. LEE and K. ZHU, Sums of products of Toeplitz and Hankel operators on the Dirichlet space, Integral Equation and Operator Theory 71 (2011), 275-302.

[13] G. MCDonALD, Fredholm properties of a class of Toeplitz operators on the ball, Indiana Univ. Math. J. 26 (1977), 567-576.

[14] K. ZHU, Spaces of holomorphic functions in the unit ball, Grad. Texts in Math. 226, Springer-Verlag, New York, 2005. 
Department of Mathematics

CHONNAM NATIONAL UNIVERSITY

GWANGJU 500-757

KOREA

E-mail address: leeyj@chonnam.ac.kr 